\title{
Isotropic-nematic phase separation in asymmetrical rod-plate mixtures
}

\author{
H. H. Wensink, G. J. Vroege, ${ }^{\text {a) }}$ and H. N. W. Lekkerkerker \\ Van 't Hoff Laboratory for Physical and Colloid Chemistry, Debye Institute, Utrecht University, \\ Padualaan 8, 3584 CH Utrecht, The Netherlands
}

(Received 4 June 2001; accepted 25 July 2001)

\begin{abstract}
Recent experiments on mixtures of rodlike and platelike colloidal particles have uncovered the phase behavior of strongly asymmetrical rod-plate mixtures. In these mixtures, in which the excluded volume of the platelets is much larger than that of the rods, an extended isotropic $(I)$-plate-rich nematic $\left(N^{-}\right)$-rod-rich nematic $\left(N^{+}\right)$triphasic equilibrium was found. In this paper, we present a theoretical underpinning for the observed phase behavior starting from the Onsager theory in which higher virial terms are incorporated by rescaling the second virial term using an extension of the Carnahan-Starling excess free energy for hard spheres (Parsons' method). We find good qualitative agreement between our results and the low concentration part of the experimental phase diagram. () 2001 American Institute of Physics. [DOI: 10.1063/1.1403686]
\end{abstract}

\section{INTRODUCTION}

Dispersions of hard rod- or platelike colloidal particles have been known to exhibit a spontaneous transition from an isotropic $(I)$ phase, in which the particles are randomly orientated to an orientationally ordered uniaxial nematic $(N)$ phase. ${ }^{1-6}$ The physical basis for understanding the phase behavior of anisometrical particles has been described in the classic work of Onsager. ${ }^{7}$ In his paper, Onsager formulated the statistical mechanics of the problem by means of a virial expansion of the free energy. He showed that the transition can be explained on the basis of repulsive interactions between the particles as embodied in the second virial term.

The phase behavior of mixtures of rodlike and platelike particles is intrinsically richer than that of rods and platelets separately. Depending on the concentration and composition, several liquid crystal phases may be formed. An issue which is currently subject to debate is the stability of the so-called biaxial nematic phase. In this liquid crystalline phase, both rods and plates are orientationally ordered but in mutually perpendicular directions. For symmetrical mixtures, i.e., in case the excluded volumes of all particle pairs are set equal, the biaxial phase appears to be a commonly established feature in many theoretical studies. ${ }^{8-13}$ Under certain conditions, the biaxial phase may, however, be unstable with respect to demixing into the separate uniaxial nematic phases, one containing predominantly rods (the $N^{+}$-phase) and platelets (the $N^{-}$-phase). ${ }^{14,15}$ In case of asymmetrical mixtures, i.e., mixtures for which the excluded volume of the platelets is much larger than that of the rods, there is strong experimental evidence of this demixing transition. Recently, van der Kooij and one of us ${ }^{16,17}$ studied the phase behavior of mixtures of hard boehmite rods (aspect-ratio 10) and gibbsite platelets (aspect-ratio 15). No evidence of a biaxial phase was found. The absence of a biaxial phase in these mixtures is consistent with the observation that the nematic phases are either strongly enriched in rods or in platelets. Since biaxial

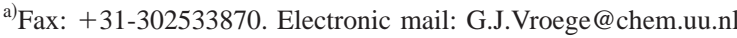

order is preferably found in well-balanced nematic phases (i.e., with approximately equal mole fractions of rods and plates $^{12}$ ) the biaxial phase is unlikely to appear in these asymmetrical mixtures.

In this paper, we use the Onsager formalism to calculate the phase diagram of asymmetrical mixtures of cylindrical rods and platelets with equal aspect-ratios and equal dimensions, so that the excluded volume of the platelets is much larger than that of the rods. In view of the aforementioned experimental results, we focus on the coexistence between the isotropic and both uniaxial nematic phases. This means that we do not take into consideration the biaxial nematic phase.

We find qualitative agreement between our diagram and the experimental one, ${ }^{16,17}$ at least in the low concentration part of the diagram. In both cases the phase behavior is initially dominated by coexistence between an isotropic phase and a plate-rich nematic $\left(N^{-}\right)$phase and, subsequently, by an extended $\left(I-N^{-}-N^{+}\right)$triphasic area. This indicates that a triphasic equilibrium at which the two uniaxial nematic phases coexist with an isotropic phase may be expected in a broad range of rod-plate compositions.

\section{THEORY}

Onsager already pointed out that the second virial approach, although valid for infinitely thin needles, cannot be justified for thin disks. The reason for this is that disks, being two-dimensional objects, have a nonzero probability of intersection and thus a finite excluded volume even at zero thickness. The relative importance of three-body interactions in terms of the ratio $B_{3} / B_{2}^{2}$ (with $B_{3}$ the third virial coefficient) has been estimated by Onsager ${ }^{7}$ at $\mathcal{O}(1)$. More accurate predictions were obtained from computer simulations, ${ }^{18}$ giving $B_{3} / B_{2}^{2} \approx 0.51$ for disks with aspect ratio $L / D=0.1$. Note that this value does not differ much from the $B_{3} / B_{2}^{2}=0.625$ for hard spheres. Even for slender rods with aspect ratios smaller than roughly 100 , the results from the second virial approximation are no longer quantitatively correct. For example, for 
spherocylinders with aspect ratio 10 the third to second virial coefficient ratio was predicted ${ }^{19}$ at $B_{3} / B_{2}^{2} \approx 0.313$, which is clearly of the same order of magnitude as the values mentioned previously.

Hence, in view of these results one must conclude that Onsager's approach of truncating the free energy after the second virial coefficient cannot be justified quantitatively for mixtures of rods and platelets with an aspect ratio of roughly 10 , which is a typical value for the experimental systems under consideration.

In order to make quantitative comparison with experiments possible we somehow have to account for the effect of higher-order correlations between particles. In the present study we use Parsons' approach ${ }^{20}$ to incorporate higher virial terms into the Onsager free energy, albeit in an approximate manner. The approach, based on the so-called decoupling approximation in which orientational and translational degrees of freedom are treated separately, comprises a rescaled form of the Carnahan-Starling free energy for hard spheres to describe the (excluded volume) interactions between the anisometrical particles. The well-known Carnahan-Starling (CS) equation of state has proven to be very accurate for hard-sphere fluids at volume fractions up to the freezing fraction $(\phi \approx 0.5)$, giving results that are almost indistinguishable from simulation predictions.

The success of the approach relies on the incorporation of higher-order interactions, albeit in an approximate manner. Lee ${ }^{21}$ has shown that Parsons' approach gave an accurate description of the isotropic to nematic transition in a system of hard ellipsoidal particles and spherocylinders as compared to MC-simulations. To give an example, the $I-N$ transition density for hard spherocylinders with aspect-ratio $L / D=5$ was calculated at a volume traction $\phi=0.400$ which practically coincides with the value predicted from Monte Carlo simulations. ${ }^{22}$ Recently, Camp and others ${ }^{13,15}$ showed that the approach could also be applied rather successfully to mixtures of rod- and platelike ellipsoids. It was shown to give improved agreement with computer simulations over the Onsager theory. The agreement with simulations was shown to be within $10 \%$.

In this section we present an analytical theory based on the approximate Gaussian trial orientation distribution function (ODF) as formulated by Odijk et al. ${ }^{23}$ which is a simplified version of the trial ODF used by Onsager. ${ }^{7}$ With the Gaussian ODF, we retain a tractable theory since it enables us to minimize the free energy analytically. On the other hand, the Gaussian ODF has proven to be very reliable in describing highly ordered nematic phases despite the fact that it is not a solution of the stationary condition as obtained from formal minimization of the free energy functional. ${ }^{24}$ For bidisperse systems of rods with different lengths, the Gaussian ODF successfully explained generic features like the fractionation effect, the widened biphasic $\operatorname{gap}^{23}$ and, somewhat later, the existence of triphasic and nematicnematic equilibria. ${ }^{25}$ A recent analysis by van $\mathrm{Roij}^{24}$ based on elaborate numerical calculations of the exact high density ODF essentially confirmed all conclusions of Ref. 25, thus emphasizing the virtues of the Gaussian approximation.
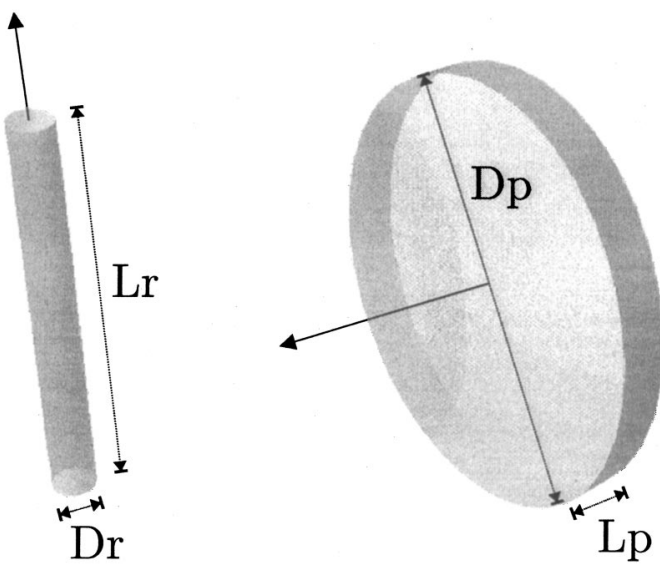

FIG. 1. Generic shape and orientation axes of the rod- and platelike particles.

In this paragraph, we will first give a description of the Onsager formalism for mixtures of rods and platelets. After that, the Parsons approach will be explained in more detail.

\section{A. Onsager theory}

We consider a binary mixture of hard rods and hard platelets in a macroscopic volume $V$. The particles involved are characterized by four parameters: the length $L_{r}$ and the diameter $D_{r}$ of the rods (with $L_{r}>D_{r}$ ) and the diameter $D_{p}$ and length (thickness) $L_{p}$ of the platelets (with $D_{p}>L_{p}$ ). The generic shape of the particles is depicted in Fig. 1. The details of the exact shape of the particles are found to be irrelevant for the general argument, provided that the particles are sufficiently anisometrical, i.e., $L_{r} / D_{r} \gg 1$ and $D_{p} / L_{p}$ $\gg 1$. When we study mixtures of rods and plates we have to realize that, in the uniaxial nematic phases, one type of particle may align along a nematic director (say the $z$-axis) while the other type tends to orient its axis randomly in a plane perpendicular to the director (the $x y$ plane). Henceforth, for the sake of definiteness, we will use subscript 1 to refer to the species oriented along the $z$ axis and subscript 2 to the species oriented in the $x y$ plane. The composition variable, $x=N_{2} /\left(N_{1}+N_{2}\right)$, thus represents the mole fraction of the species with preferred perpendicular orientation. Hence, $x$ represents the mole fraction of platelets in case of $I-N^{+}$coexistence and the fraction of rods in case of $I-N^{-}$ coexistence.

Onsager described the nematic phase of a dilute solution of anisometrical particles in terms of the orientational distribution function (ODF), $f_{j}(\theta)$, representing the distribution of the angles between the axis of the particle and the nematic director. The ODF must be normalized according to $\int f_{j}(\Omega) d \Omega \equiv 1$, where $\Omega$ is the solid angle of the particle's normal vector. In the isotropic state, all orientations are equally probable which implies $f_{\text {iso }} \equiv 1 / 4 \pi$. In the present study, we use Gaussian trial ODFs with variational parameters $\alpha_{j}$ to describe the angular distribution of the particles in the nematic phase. Hence, we write for particles oriented along the director 
$f_{1}(\theta) \equiv\left\{\begin{array}{ll}\frac{\alpha_{1}}{4 \pi} \exp \left[-\frac{1}{2} \alpha_{1} \theta^{2}\right] & 0 \leqslant \theta \leqslant \frac{\pi}{2} \\ \frac{\alpha_{1}}{4 \pi} \exp \left[-\frac{1}{2} \alpha_{1}(\pi-\theta)^{2}\right] & \frac{\pi}{2} \leqslant \theta \leqslant \pi\end{array}\right.$,

and for particles oriented perpendicular to the nematic director

$f_{2}(\theta) \equiv \sqrt{\frac{\alpha_{2}}{(2 \pi)^{3}}} \exp \left[-\frac{1}{2} \alpha_{2}\left(\frac{\pi}{2}-\theta\right)^{2}\right] \quad 0 \leqslant \theta \leqslant \pi$.

Note that $f_{1}(\theta)$ is peaked around the nematic director $(\theta$ $=0$ and $\theta=\pi)$ whereas $f_{2}(\theta)$ attains its maximum value in the plane perpendicular to the nematic director $(\theta=\pi / 2)$. The formation of an isotropic state (with $f_{j}$ constant) or a nematic state (with $f_{j}$ a peaked distribution) is caused by a competition between orientational entropy (favoring the isotropic state) and excluded volume entropy (favoring the nematic state). Onsager ${ }^{7}$ defined $\sigma_{j}$ as a measure for the (negative of the) orientational entropy

$$
\sigma_{j} \equiv \int f_{j}(\theta) \ln \left[4 \pi f_{j}(\theta)\right] d \Omega, \quad j=1,2,
$$

which has its minimum $\left(\sigma_{j}=0\right)$ in the isotropic state but increases as the orientational entropy decreases. An important implication of using Gaussian trial ODFs is that it enables us to calculate $\sigma_{j}$ analytically. For the nematic state we obtain after substituting Eqs. (1), (2) and straightforward integration

$$
\begin{aligned}
& \sigma_{1} \sim \ln \alpha_{1}-1, \\
& \sigma_{2} \sim \frac{1}{2}\left(\ln \alpha_{2}+\ln \frac{2}{\pi}-1\right) .
\end{aligned}
$$

In the second virial approximation, the interactions between hard particles may be expressed as an excluded volume entropy depending on the excluded volume between two particles. Onsager gives us the following expression for the excluded volume between two platelets (i.e., circular disks), a platelet and a (cylindrical) rod and two rods as a function of the angle $\gamma$ between the particles' axes

$$
\begin{aligned}
& v_{\mathrm{excl}}^{\mathrm{pp}}(\gamma)=\frac{\pi}{2} D_{p}^{3}|\sin \gamma|+\mathcal{O}\left(D_{p}^{2} L_{p}\right), \\
& v_{\mathrm{excl}}^{\mathrm{rp}}(\gamma)=\frac{\pi}{4} L_{r} D_{p}^{2}|\cos \gamma|+\mathcal{O}\left(L_{r} D_{p} D_{r}\right), \\
& v_{\mathrm{excl}}^{\mathrm{rr}}(\gamma)=2 L_{r}^{2} D_{r}|\sin \gamma|+\mathcal{O}\left(L_{r} D_{r}^{2}\right) .
\end{aligned}
$$

Note that we restrict ourselves to the leading order contributions, which is justified for sufficiently anisometrical particles. Using the isotropic averages $\langle\langle\sin \gamma\rangle\rangle_{\text {iso }}=\pi / 4$ and $\langle\langle\cos \gamma\rangle\rangle_{\text {iso }}=1 / 2$ we obtain the average excluded volume between two randomly oriented particles in the isotropic phase

$$
\begin{aligned}
& v_{\mathrm{excl}, \mathrm{iso}}^{\mathrm{pp}}=\frac{\pi^{2}}{8} D_{p}^{3}, \quad v_{\mathrm{exc}, \text { iso }}^{\mathrm{rp}}=\frac{\pi}{8} L_{r} D_{p}^{2}, \\
& v_{\mathrm{excl}, \mathrm{iso}}^{\mathrm{rr}}=\frac{\pi}{2} L_{r}^{2} D_{r} .
\end{aligned}
$$

A measure for the average excluded-volume interaction between two particles is given by the average of its angular dependence, defined as follows:

$$
\rho_{j k} \equiv \iint \frac{v_{\text {excl }}^{j k}(\gamma)}{v_{\text {excl,iso }}^{j k}} f_{j}(\theta) f_{k}\left(\theta^{\prime}\right) d \Omega d \Omega^{\prime}
$$

which implies $\rho_{j k} \equiv 1$ for the isotropic phase. For the nematic phase, we must substitute Eq. (6). Hence, for two rods or platelets orientated either along or perpendicular to the nematic director this integral reads

$\rho_{j j}=\frac{4}{\pi} \iint\left|\sin \gamma\left(\Omega, \Omega^{\prime}\right)\right| f_{j}(\theta) f_{j}\left(\theta^{\prime}\right) d \Omega d \Omega^{\prime}, \quad j=1,2$.

Similarly, for a rod and a plate with mutually perpendicular orientations we have

$$
\rho_{12}=2 \iint\left|\cos \gamma\left(\Omega, \Omega^{\prime}\right)\right| f_{1}(\theta) f_{2}\left(\theta^{\prime}\right) d \Omega d \Omega^{\prime} .
$$

Unlike Eq. (3), the excluded volume integrals cannot be calculated straightforwardly since the integrands depend on the interparticle angle $\gamma\left(\Omega, \Omega^{\prime}\right)$. However, we can make headway by performing an asymptotic expansion of the integrals, valid for small angles $\theta$ and $\psi=\pi / 2-\theta^{\prime}$, implying that the particles' axes in general marginally deviate from the nematic director or the $x y$ plane. Obviously, the asymptotic expansion is only justified for large $\alpha_{j}$, i.e., the Gaussian ODFs must be sharply peaked around their maximum values. Retaining only the leading order terms of the asymptotic expansions, we readily obtain for $\rho_{11}$ (in case of orientation along the director)

$$
\rho_{11} \sim \frac{4}{\sqrt{\pi \alpha_{1}}}+\mathcal{O}\left(\alpha_{1}^{-3 / 2}\right),
$$

which was already found by Odijk et al. ${ }^{23}$ The excluded volume integral for a rod and a plate with mutual perpendicular orientations $\rho_{12}$ requires a bit more effort (see Appendix A for details). The result is as follows:

$$
\rho_{12} \sim \sqrt{\frac{8}{\pi}\left(\frac{1}{\alpha_{1}}+\frac{1}{\alpha_{2}}\right)}+\mathcal{O}\left(\alpha_{1}^{-3 / 2}, \alpha_{2}^{-3 / 2}\right) .
$$

Remarkably, the leading order term is equal to the one obtained for rods with two different lengths. ${ }^{23}$ Finally, for the averaged excluded volume between two particles preferentially oriented in the $x y$ plane we can write

$$
\rho_{22} \sim \rho_{22,0}\left[1+\mathcal{F}\left(\alpha_{2}\right)\right],
$$

where the leading order term $\rho_{22,0}$ is simply the average excluded volume between two particles $j$ randomly orientated in the $x y$ plane $\left(\psi=\psi^{\prime}=0\right)$ relative to $v_{\text {excl,iso }}^{22}$

$$
\rho_{22,0}=(4 / \pi)\left[\int_{0}^{\pi} d \gamma\right]^{-1} \int_{0}^{\pi} d \gamma \sin \gamma=\frac{8}{\pi^{2}} .
$$

The $\alpha_{2}$-depending correction term $\mathcal{F}$ is rather difficult to obtain. It reads 


$$
\mathcal{F}\left(\alpha_{2}\right) \sim \frac{1}{\alpha_{2}}\left(\frac{1}{2} \ln \alpha_{2}+K\right)+\mathcal{O}\left(\alpha_{2}^{-2} \ln \alpha_{2}\right),
$$

where $K=\ln 4+\frac{1}{2} \gamma_{E}-\frac{3}{2}$ and $\gamma_{E} \approx 0.577$ Euler's constant. We will elaborate on its derivation in Appendix A. It is important to note that $\mathcal{F}$ scales as $\alpha_{2}^{-1} \ln \alpha_{2}$ whereas $\rho_{11}$ and $\rho_{12}$ both scale as $\alpha^{-1 / 2}$. So, for large $\alpha_{j}, \mathcal{F}$ leads to a small contribution compared to $\rho_{11}$ and $\rho_{12}$.

The total Helmholtz free energy $F$ of the rod-plate mixture (in units $k_{B} T$ per particle) can now be expressed in terms of the parameters $\sigma_{j}$ and $\rho_{j k}$

$$
\begin{aligned}
\frac{\beta F}{N} \simeq & \operatorname{cst}+\ln c-1+(1-x) \ln (1-x)+x \ln x \\
& +(1-x) \sigma_{1}+x \sigma_{2} \\
& +c\left[(1-x)^{2} \rho_{11}+2 x(1-x) q_{12} \rho_{12}+x^{2} q_{22} \rho_{22}\right],
\end{aligned}
$$

where $\beta=\left(k_{B} T\right)^{-1}\left(k_{B}\right.$ and $T$ have their usual meaning of Boltzmann's constant and absolute temperature). The constant cst includes terms independent of the mole fraction and concentration. Furthermore, $c$ is the total number density of particles rendered dimensionless by relating it to $v_{\text {excl,iso }}^{11}$ via

$$
c=\frac{1}{2} v_{\text {excl,iso }}^{11} \frac{N_{1}+N_{2}}{V}=\left\{\begin{array}{cc}
\frac{\pi}{4} L_{r}^{2} D_{r} \frac{N}{V} & N^{+} \text {-phase } \\
\frac{\pi^{2}}{16} D_{p}^{3} \frac{N}{V} & N^{-} \text {-phase }
\end{array} .\right.
$$

The last term in the free energy Eq. (16) can be identified as a (dimensionless) second virial coefficient $\widetilde{B}_{2}$ multiplied by the dimensionless concentration $c$. Note that $c \widetilde{B}_{2}$ constitutes the excess part of the free energy which accounts for the interactions between the hard particles. Using the expressions for $\sigma_{j}$ and $\rho_{j k}$ in Eq. (16) and minimizing with respect to $\alpha_{1}$ and $\alpha_{2}$ yields

$$
\begin{aligned}
& \alpha_{1}^{1 / 2}=2 \pi^{-1 / 2}\left[(1-x)+2^{1 / 2} x q_{12} h(Q)\right] c, \\
& \alpha_{2}^{1 / 2}=\left[2^{5 / 2} \pi^{-1 / 2}(1-x) q_{12} g(Q)-\mathcal{H}\left(x, \alpha_{2}\right)\right] c,
\end{aligned}
$$

with the definitions

$$
\begin{aligned}
& Q \equiv \alpha_{2} / \alpha_{1}, \\
& h(Q) \equiv Q^{1 / 2} g(Q) \equiv\left(\frac{Q}{Q+1}\right)^{1 / 2},
\end{aligned}
$$

and

$$
q_{12}=v_{\text {excl,iso }}^{12} / v_{\text {excl,iso }}^{11}, \quad q_{22}=v_{\text {excl,iso }}^{22} / v_{\text {excl,iso }}^{11} .
$$

Furthermore, $\mathcal{H}$ is the contribution arising from $\mathcal{F}\left(\alpha_{2}\right)$

$$
\mathcal{H}\left(x, \alpha_{2}\right)=\frac{8}{\pi^{2}} x q_{22} \alpha_{2}^{-1 / 2}\left[1-2 K-\ln \alpha_{2}\right],
$$

which is small for large $\alpha_{2}$. To simplify matters, we set $\mathcal{F}$ (and $\mathcal{H}$ ) equal to 0 for the moment, so that $\rho_{22}=\rho_{22,0}$ $=8 / \pi^{2}$. Henceforth, this will be referred to as the zeroth order problem, denoted by subscripts 0 . Within this approximation, it is possible to combine both minimization equations in order to obtain an expression only involving the ratio of both $\alpha$ 's. Taking the ratio of Eqs. (18) and (19) gives

$$
Q_{0}^{1 / 2}=\frac{2^{3 / 2}(1-x) q_{12} g\left(Q_{0}\right)}{(1-x)+2^{1 / 2} x q_{12} h\left(Q_{0}\right)},
$$

which is an implicit equation for $Q_{0}$ only containing the mole fraction $x$ and not the concentration $c$, so $Q_{0}$ $=Q_{0}(x)$. A similar equation was obtained by Odijk et al. for bidisperse rods. Fortunately, Eq. (24) can be solved analytically, unlike the one obtained for bidisperse rods. After rearranging terms, Eq. (24) can be rewritten as a simple quadratic equation in $Q_{0}$, which is easily solvable. In practice, it is convenient to rewrite the excluded volume terms $\rho_{j k}$ in terms of $Q_{0}, x$ and $c$ using the minimization equations (18), (19) and then substitute $Q_{0}(x)$ as found from Eq. (24). The free energy of the uniaxial nematic phase is then written explicitly in terms of the composition and dimensionless concentration of the phase.

To locate phase transitions, we must know the osmotic pressure and chemical potential of both species. These are easily calculated as derivatives of the free energy. In the nematic phase (denoted by subscript $n$ ) we get in dimensionless notation

$$
\begin{aligned}
\widetilde{\Pi}_{n} & \equiv-\frac{1}{2} v_{\text {excl,iso }}^{11} \beta\left(\frac{\partial F}{\partial V}\right)_{N_{1}, N_{2}, T} \sim(3-x) c+\frac{8}{\pi^{2}} q_{22} x^{2} c^{2} \\
\tilde{\mu}_{1, n} & \equiv \beta\left(\frac{\partial F}{\partial N_{1}}\right)_{N_{2}, V, T} \\
& \sim \ln c+\ln (1-x)+\sigma_{1}+2 c\left[(1-x) \rho_{11}+x q_{12} \rho_{12}\right] \\
\tilde{\mu}_{2, n} & \equiv \beta\left(\frac{\partial F}{\partial N_{2}}\right)_{N_{1}, V, T} \\
& \sim \ln c+\ln x+\sigma_{2}+2 c\left[(1-x) q_{12} \rho_{12}+x q_{22} \rho_{22}\right] .
\end{aligned}
$$

Similarly, we obtain for the isotropic phase (denoted by subscript $i$ ) using the isotropic values $\sigma_{j} \equiv 0$ and $\rho_{j k} \equiv 1$

$$
\begin{aligned}
& \widetilde{\Pi}_{i} \simeq c+c^{2} \widetilde{B}_{2, i}, \\
& \tilde{\mu}_{1, i} \simeq \ln c+\ln (1-x)+2 c\left[(1-x)+x q_{12}\right], \\
& \tilde{\mu}_{2, i} \simeq \ln c+\ln x+2 c\left[(1-x) q_{12}+x q_{22}\right],
\end{aligned}
$$

where $\widetilde{B}_{2, i}=\left[(1-x)^{2}+2 x(1-x) q_{12}+x^{2} q_{22}\right]$ is the dimensionless second virial coefficient for the isotropic phase.

When we use Eqs. (15) and (23), the implicit equation for the nematic phase becomes much more difficult. In fact, it can be shown that $Q$ will be dependent on the concentration as well, which considerably complicates our further analysis. We will therefore not attempt to solve the full equation. To make headway, we will account for $\mathcal{F}\left(\alpha_{2}\right)$ in a perturbative way. Since $\mathcal{F}$ is only a small contribution, parameters $\alpha_{j}, \sigma_{j}$ and $\rho_{j k}$ obtained from the minimization of the free energy will only marginally differ from the ones obtained for $\mathcal{F}=0$. Hence, $\mathcal{F}$ can be considered as a perturba- 
tion to the parameters obtained from the zeroth order problem. The approach will be discussed in Appendix B.

As mentioned in the introductory part, although Onsager's second virial approximation works well for sufficiently elongated rods it does not give quantitative results for platelike particles. Therefore, in case of rod-plate mixtures, manybody interactions involving platelets will undoubtedly play a role in the regime where nematic phases appear. In order to make quantitative progress, we have to somehow account for the effect of higher virial terms. A method which has proved to be useful up to now is to rescale the excess part of the Onsager free energy using a modified form of the CarnahanStarling excess free energy for hard spheres. This approach, known as the Parsons approach, will be discussed in the next section.

\section{B. The Parsons approach}

The Parsons approach involves an expression of the excess free energy in terms of the semi-empirical CarnahanStarling free energy for hard spheres ${ }^{26}$

$$
f_{\mathrm{CS}}(\phi)=\frac{\beta F_{\mathrm{CS}}^{\mathrm{ex}}}{N}=\frac{\phi(4-3 \phi)}{(1-\phi)^{2}},
$$

where $\phi$ is the volume fraction of hard spheres. For a onecomponent system of hard anisometrical particles this free energy is multiplied by the prefactor $\left\langle\left\langle v_{\text {excl }}\right\rangle\right\rangle / 8 v_{0}$ with $v_{0}$ the particle volume and $\left\langle\left\langle v_{\text {excl }}\right\rangle\right\rangle$ the average excluded volume. Note that $\left\langle\left\langle v_{\text {excl }}\right\rangle\right\rangle / 8 v_{0}=1$, in the case of hard spheres. For binary mixtures of anisometrical particles, the prefactor may be rewritten as $\left\langle\left\langle\bar{v}_{\text {excl }}\right\rangle\right\rangle / \bar{v}_{0}$ in terms of the following mole fraction averages ${ }^{13}$

$$
\begin{aligned}
& \left\langle\left\langle\bar{v}_{\text {excl }}\right\rangle\right\rangle=v_{\text {excl,iso }}^{11}\left[(1-x)^{2} \rho_{11}+2 x(1-x) q_{12} \rho_{12}+x^{2} q_{22} \rho_{22}\right] \\
& \quad=v_{\text {excl,iso }}^{11} \widetilde{B}_{2}, \\
& \bar{v}_{0}=(1-x) v_{0,1}+x v_{0,2}, \\
& \phi=(1-x) \phi_{1}+x \phi_{2},
\end{aligned}
$$

where $\phi$ is the total volume fraction of particles. Quantitatively, the results from this approach compare reasonably well with computer simulations as was shown by Camp et al. ${ }^{13,15}$ Having established this, we can write the excess free energy within the Parsons approach as follows:

$$
\begin{aligned}
\frac{\beta F^{\mathrm{ex}}}{N} & =c\left[(1-x)^{2} \rho_{11}+2 x(1-x) q_{12} \rho_{12}+x^{2} q_{22} \rho_{22}\right] \widetilde{f}_{\mathrm{CS}}(\phi) \\
& =c \widetilde{B}_{2} \widetilde{f}_{\mathrm{CS}}(\phi)
\end{aligned}
$$

where $\widetilde{f}_{\mathrm{CS}}(\phi)=f_{\mathrm{CS}}(\phi) / 4 \phi$. Replacing the excess free energy $c \widetilde{B}_{2}$ in Eq. (16) by Eq. (33) gives us the OnsagerParsons free energy for a binary mixture of hard rods and platelets. Minimization with respect to $\alpha_{j}$ now yields (for the zeroth order problem, $\mathcal{F}=0$ )

$$
\begin{aligned}
& \alpha_{1}^{1 / 2}=2 \pi^{-1 / 2}\left[(1-x)+2^{1 / 2} x q_{12} h\left(Q_{0}\right)\right] c \widetilde{f}_{\mathrm{CS}}(\phi), \\
& \alpha_{2}^{1 / 2}=2^{5 / 2} \pi^{-1 / 2} q_{12}(1-x) g\left(Q_{0}\right) c \widetilde{f}_{\mathrm{CS}}(\phi) .
\end{aligned}
$$

Note that the implicit equation in $Q_{0}\left(=\alpha_{2} / \alpha_{1}\right)$ is left unchanged since the concentration dependent part $c \widetilde{f}_{\mathrm{CS}}(\phi)$ cancels. The total volume fraction $\phi$ of rods and platelets is related to the dimensionless concentration $c$ and the mole fraction $x$ via

$$
\phi(c, x)=2 c\left[(1-x) \frac{v_{0,1}}{v_{\mathrm{ex}, \mathrm{iso}}^{11}}+x \frac{v_{0,2}}{v_{\mathrm{ex}, \mathrm{iso}}^{11}}\right] .
$$

It is now straightforward to recalculate the osmotic pressure and chemical potentials by substituting Eq. (35) and $Q_{0}(x)$ [from Eq. (24)] in the Onsager-Parsons free energy and taking the adequate derivatives. However, these expressions are rather intricate since additional derivatives of $\widetilde{f}_{\mathrm{CS}}$ with respect to $c$ and $x$ are now involved. Within the Parsons approach, the osmotic pressure of the nematic phase (denoted by $n$ ) reads

$$
\widetilde{\Pi}_{n}^{P} \sim c_{n}+\left[\left(2-x_{n}\right) c_{n}+\frac{8}{\pi^{2}} c_{n}^{2} x_{n}^{2} q_{22} \widetilde{f}_{\mathrm{CS}}\right]\left[1+\frac{\partial \ln \widetilde{f}_{\mathrm{CS}}}{\partial \ln c}\right],
$$

and the chemical potentials

$$
\begin{aligned}
\tilde{\mu}_{1, n}^{P}= & \tilde{\mu}_{1, n}+2 \ln \widetilde{f}_{\mathrm{CS}}+\left[(2-x)+c \widetilde{f}_{\mathrm{CS}} x^{2} q_{22} \frac{8}{\pi^{2}}\right] \\
& \times \frac{\partial \ln \widetilde{f}_{\mathrm{CS}}}{\partial(1-x)}, \\
\tilde{\mu}_{2, n}^{P}= & \tilde{\mu}_{2, n}+\ln \widetilde{f}_{\mathrm{CS}}+\left[(2-x)+c \widetilde{f}_{\mathrm{CS}} x^{2} q_{22} \frac{8}{\pi^{2}}\right] \frac{\partial \ln \widetilde{f}_{\mathrm{CS}}}{\partial x} .
\end{aligned}
$$

Recall that these expressions only hold for the zeroth order problem $\left(\rho_{22}=\rho_{22,0}\right)$. When using the full expression for $\rho_{22}$ Eq. (13), additional terms arising from the perturbation theory must be included (see Appendix B). These contributions are omitted here for the sake of simplicity, but can be obtained straightforwardly. For the isotropic phase (denoted by $i$ ) we obtain

$$
\begin{aligned}
\widetilde{\Pi}_{i}^{P}= & c_{i}+c_{i}^{2} \widetilde{f}_{\mathrm{CS}} \widetilde{B}_{2, i}\left[1+c_{i} \frac{\partial \ln \widetilde{f}_{\mathrm{CS}}}{\partial c_{i}}\right], \\
\widetilde{\mu}_{1, i}^{P}= & \ln c_{i}+\ln \left(1-x_{i}\right) \\
& +c_{i} \widetilde{f}_{\mathrm{CS}}\left[\widetilde{B}_{2, i} \frac{\partial \ln \widetilde{f}_{\mathrm{CS}}}{\partial\left(1-x_{i}\right)}+2\left(1-x_{i}\right)+2 x_{i} q_{12}\right], \\
\tilde{\mu}_{2, i}^{P}= & \ln c_{i}+\ln x_{i} \\
& +c_{i} \widetilde{f}_{\mathrm{CS}}\left[\widetilde{B}_{2, i} \frac{\partial \ln \widetilde{f}_{\mathrm{CS}}}{\partial x_{i}}+2\left(1-x_{i}\right) q_{12}+2 x_{i} q_{22}\right] .
\end{aligned}
$$

\section{PHASE DIAGRAMS}

We can now construct the phase diagrams by imposing the standard conditions of equal osmotic pressure and chemical potentials in the coexisting phases, using the expressions 


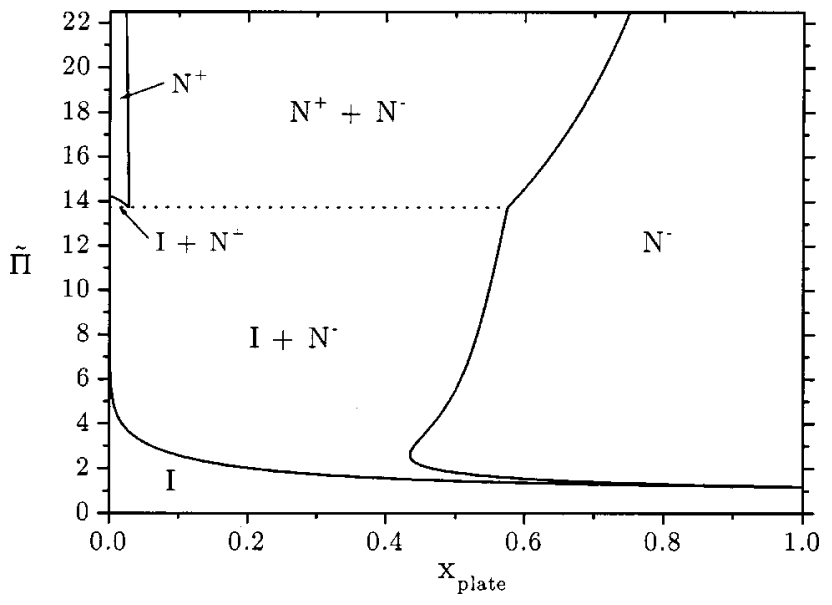

FIG. 2. Phase diagram as calculated from the perturbation theory in the osmotic pressure-composition $(\widetilde{\Pi}-x)$ plane for a mixture of rods and plates $\left(L_{r} / D_{r}=D_{p} / L_{p}=15\right)$ with equal dimensions, i.e., $L_{r}=D_{p}$ and $D_{r}=L_{p}$. Note the reentrant phenomenon near $x_{\text {plate }}=0.44$.

from the Onsager-Parsons free energy and adding the perturbative contributions. The phase diagrams were constructed as follows. The $I-N^{-}$and $I-N^{+}$coexistence curves were calculated separately using $\left(q_{11}, q_{22}\right)=\left(q_{p p}, q_{r r}\right)$ and $\left(q_{11}, q_{22}\right)=\left(q_{r r}, q_{p p}\right)$, respectively, and solving the coexistence equations iteratively. A triphasic equilibrium was located at the intersection point (triple osmotic pressure) of the two isotropic branches. At the triple pressure, an isotropic phase coexists with two nematic phases, each with different mole fraction and number density. The location of the triple point (in terms of $x$ and $c$ ) could subsequently be used as a starting point for the calculation of the $N^{+}-N^{-}$coexistence branches.

To give a graphic representation of the results, a $\widetilde{\Pi}-x$ diagram is shown in Fig. 2 and a diagram in terms of volume fractions in Fig. 3. The volume fraction representation may be more convenient from an experimental standpoint. The volume fractions are obtained straightforwardly from Eq. (35). The tie lines are given by horizontal lines in Fig. 2 because of equality of osmotic pressure. In the volume frac-

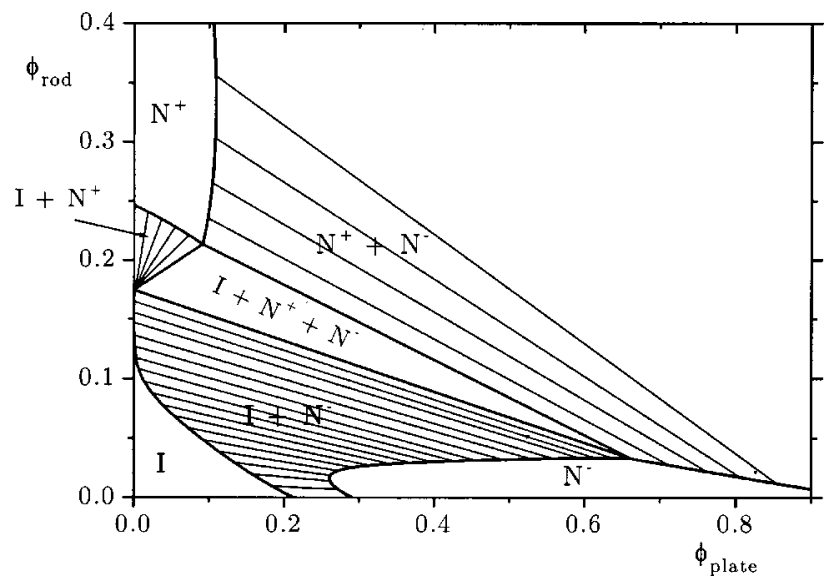

FIG. 3. Phase diagram in the $\phi_{\text {rod }}-\phi_{\text {plate }}$ representation corresponding to Fig. 2. Thick lines indicate phase boundaries; thin lines represent tie lines connecting coexisting phases. tion representation these tie lines may be shown to form straight lines. ${ }^{27}$ In the latter case, we can also draw dilution lines, i.e., straight lines radiating from the origin, along which the overall mole fraction $x$ of the parent system remains constant. Obviously, in the $\widetilde{\Pi}-x$ representation these dilution lines run vertically.

To facilitate comparison with experimental results we have matched the dimensions of the particles under consideration to the average size of the gibbsite particles used in experiment. ${ }^{17}$ Accordingly, we have chosen equal aspect ratios for the rods and platelets, i.e., $L_{r} / D_{r}=D_{p} / L_{p}=15$. Furthermore, the long and short dimensions of the particles were chosen to be equal, so that $L_{r}=D_{p}$ and $D_{r}=L_{p}$. The ratios of the excluded volumes are then given by

$$
q_{r r}=1, \quad q_{r p}=15 / 4, \quad q_{p p}=15 \pi / 4,
$$

indicating that the isotropic excluded volume of two platelets is almost 12 times larger than the excluded volume of two rods. The resulting mixture is thus strongly asymmetrical $\left(v_{\text {excl }}^{r r} \ll v_{\text {excl }}^{r p} \ll v_{\text {excl }}^{p p}\right)$.

Several features are notable from Figs. 2 and 3. First, there is a reentrant transition at mole fractions $x_{\mathrm{p}}$ between 0.44 and 0.58 . Experimentally, this would imply that a dilute sample containing $50 \%$ platelets undergoes numerous phase transitions upon concentrating, going from the isotropic state $I$ to $I+N^{-}, N^{-}, I+N^{-}, I+N^{-}+N^{+}$and finally $N^{-}+N^{+}$. A similar reentrant transition was found in binary mixtures of rods with different lengths. ${ }^{28,23}$ Furthermore, the triphasic equilibrium, represented by a triple line in the $\widetilde{\Pi}-x$ representation, clearly manifests itself in the volume fraction representation as a triphasic triangle which covers a fair part of the phase diagram. Accordingly, a large range of compositions will pass through the three phase area. It appears that a very small mole fraction of platelets in the isotropic phase already leads to a three phase equilibrium upon increasing the overall concentration.

We also investigate the phase behavior of a mixture with reduced asymmetry, i.e., the differences in excluded volume are chosen to be less extreme. For that, we consider a mixture with aspect ratio $L_{r} / D_{r}=D_{p} / L_{p}=50$ and size ratio $L_{r}$ $=2 D_{p}, D_{r}=2 L_{p}$. So, again, both species are equally anisometrical but the size of the platelets is now reduced to half the size of the rods. This leads to the following excluded volume ratios

$$
q_{r r}=1, \quad q_{r p} \approx 3.13, \quad q_{p p} \approx 4.91 .
$$

Note that $q_{p p}$ is reduced by a factor 2.4 compared to Eq. (40). Yet, the excluded volume of two (randomly oriented) platelets is still about five times larger than that of two rods. The corresponding phase diagrams are shown in Figs. 4 and 5 .

Again, we observe a reentrant transition around $x_{\mathrm{p}}$ $=0.5$, albeit less prominent than in Fig. 2. A remarkable difference with Fig. 2, however, is the presence of an azeotropic point in the plate-rich part (the 'tail' part) of the $\widetilde{\Pi}-x$ diagram [see Fig. 4(b)]. The azeotropic point is located at mole fraction $x_{\mathrm{p}}=0.718$. Accordingly, a mixture containing $71.8 \%$ platelets (with $L_{r}=2 D_{p}$ ) does not fractionate during 

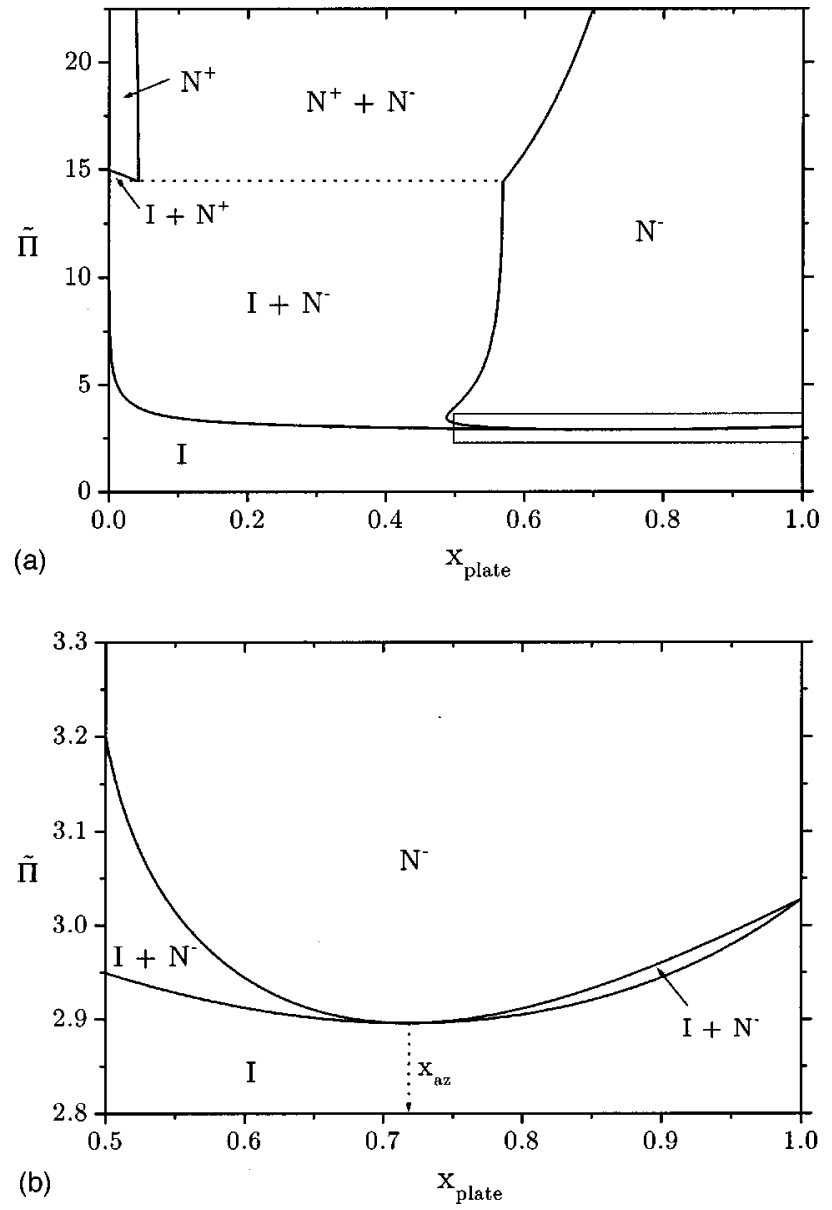

FIG. 4. (a) Phase diagram as calculated from the perturbation theory in the $\widetilde{\Pi}-x$ plane for $L_{r} / D_{r}=D_{p} / L_{p}=50$. The platelets are half the size of the rods $\left(L_{r}=2 D_{p}\right.$ and $\left.D_{r}=2 L_{p}\right)$. A reentrant phenomenon near $x_{\text {plate }}=0.5$ is evident. (b) Magnification of the 'tail part' of the diagram. Note the azeotropic point at $x_{\mathrm{az}}=0.718$.

phase separation, i.e., the coexisting $I$ and $N^{-}$daughter phases have the same mole fraction as the parent sample.

An azeotropic point always corresponds to an extremum in the osmotic pressure as a function of the mole fraction. In

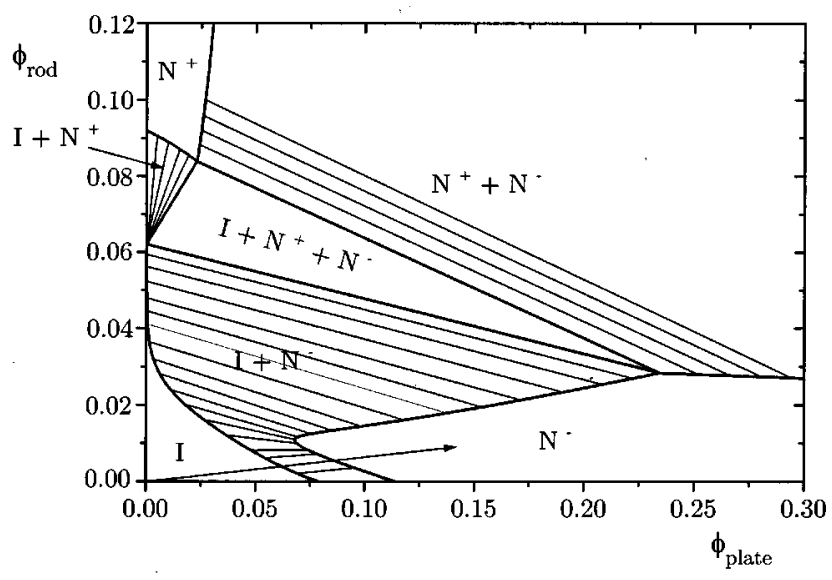

FIG. 5. Phase diagram in the $\phi_{\text {rod }}-\phi_{\text {plate }}$ representation corresponding to Fig. 4(a). Thick lines indicate phase boundaries; thin lines indicate represent tie lines connecting coexisting phases. A particular dilution line corresponding to the azeotropic point is drawn. Note that the associated tie line coincides with the azeotropic dilution line. this case, there is a minimum in the osmotic pressure at $x_{\mathrm{p}}$ $=0.718$, as can be seen from Fig. 4(b). By recalculating the phase diagram for various ratios $L_{r} / D_{p}$ on the interval 1 $<L_{r} / D_{p}<2$ one can show that the azeotropic point (and hence the minimum in $\widetilde{\Pi}$ ) shifts toward higher mole fractions as the ratio $L_{r} / D_{p}$ is lowered. Obviously, noting its absence in Fig. 3, the azeotropic point must leave the scene at some point on this interval. This, so-called "critical" ratio can be determined by calculating, for instance, the initial fractionation, which we define as $x_{p}^{I}-x_{p}^{N}$ for $x_{p}$ infinitesimally close to 1 , as a function of the ratio $L_{r} / D_{p}$. At the critical ratio, the azeotropic point is located at $x_{p}=1$ and the initial fractionation is thus equal to zero. Note that the initial fractionation is positive in case of an azeotropic point since the coexistence pressure decreases initially at $x_{p}=1$. In case of no azeotropic point, the coexistence pressure increases upon lowering $x_{p}$ and the initial fractionation is negative. The critical ratio $L_{r} / D_{p}$ was found to be 1.54 , independent of the aspect ratio $L_{r} / D_{r}=D_{p} / L_{p}$. Hence, in case of equal aspect ratios, we may expect an azeotropic point only if the rods are sufficiently larger than the platelets (i.e., $L_{r}$ $\left.>1.54 D_{p}\right)$.

\section{DISCUSSION}

We have constructed the phase diagram of a rod-plate mixture with strongly asymmetric excluded volumes $\left(v_{\mathrm{ex}}^{\text {plate }}\right.$ $\left.\gg v_{\mathrm{ex}}^{\text {rod }}\right)$ using Onsager's second virial approximation. In our approach, we used Gaussian ODFs with adjustable parameters $\alpha_{j}$ to describe the distribution of angles in the uniaxial nematic phases. To account for the effect of higher virial terms, we have rescaled the excess free energy according to Parsons' method.

In the present calculations, we have set up a perturbation theory to account for the $\ln \alpha_{2} / \alpha_{2}$-term Eq. (15) in the asymptotic expansion of the excluded volume integral $\rho_{22}$. To check the validity of this theory, we may compare it with a full numerical approach, in which the minimization equations are solved numerically along with the coexistence equations. As an example, we have depicted some $I-N^{-}$ binodals obtained from several approaches in Fig. 6. The differences between the approaches seem to become more pronounced at low aspect ratios. First, agreement between the perturbation theory and the full numerical solution is very good. In fact, it remains surprisingly good even at low aspect ratios (below 15) whereas at high ratios $(>20)$ the curves almost become indistinguishable. Second, the zeroth order approximation clearly starts to deviate from the other curves as the aspect ratio is lowered. Crucially, the zeroth order approximation (i.e., retaining only the leading order constant in $\rho_{22}$ ) completely breaks down at low aspect ratios (say, $L_{r} / D_{r}<18$ ) giving unphysical binodals. This breakdown is not encountered within the perturbation theory or the numerical approach. Therefore, we conclude that the second order contribution $\mathcal{F}$ to $\rho_{22}$ is an essential ingredient in our calculations, since it enables us to calculate the phase behavior of mixtures with aspect ratios comparable to that of experimental systems (i.e., $L_{r} / D_{r}$ roughly between 10 and 15). 


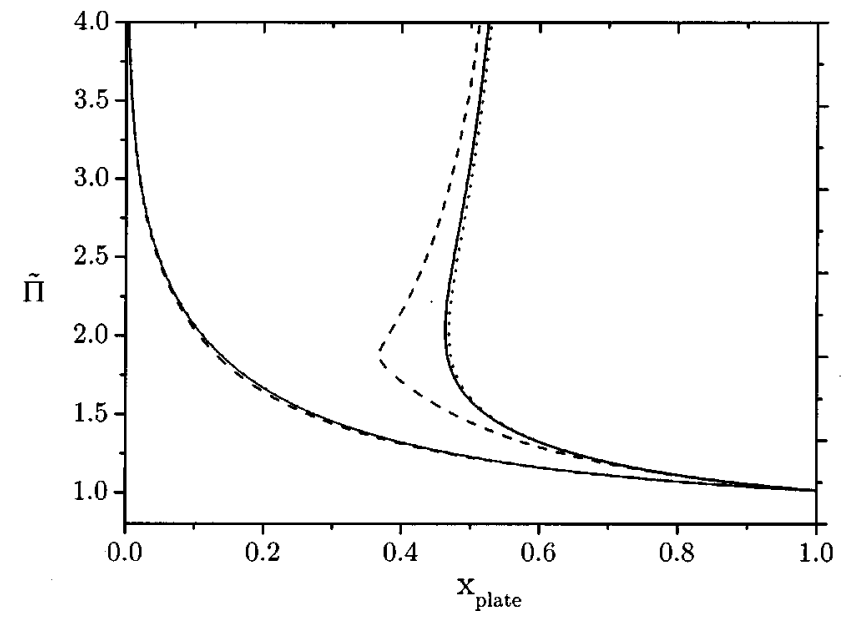

FIG. 6. $I-N^{-}$binodals for $L_{r} / D_{r}=D_{p} / L_{p}=18$ and $L_{r}=D_{p}, D_{r}=L_{p}$ as calculated from several approaches; zeroth order approximation (dashed line), perturbation theory (dotted line) and full numerical approach (solid line).

Let us now compare our calculated phase diagram with the experimental one as constructed in Refs. 16 and 17 . We have depicted the experimental diagram in Fig. 7. This diagram can be compared directly with our volume fraction representation in Fig. 3. From a qualitative point of view, both diagrams agree very well. The general topology of both diagrams is very similar, at least in the low concentration part. A notable feature is that the phase behavior is largely dominated by coexistence between the isotropic phase and the plate-rich nematic $\left(N^{-}\right)$phase whereas coexistence between $I$ and the rod-rich nematic $\left(N^{+}\right)$phase is only evident in a small area close to the rod axis. This is a manifestation of the asymmetrical nature of the mixture, i.e., the excluded volume of the platelets is much larger than the excluded volume of the rods. Note that the fractionation between the $I$ and $N^{-}$ phases is also much more pronounced, particularly at high osmotic pressures, than for $I-N^{+}$coexistence. Finally, an $I-N^{-}-N^{+}$triphasic area covering a significant part of the phase diagram is present in both theory and experiment. A generic feature that does not seem to appear in the experimental diagram is the reentrant transition. However, a de-

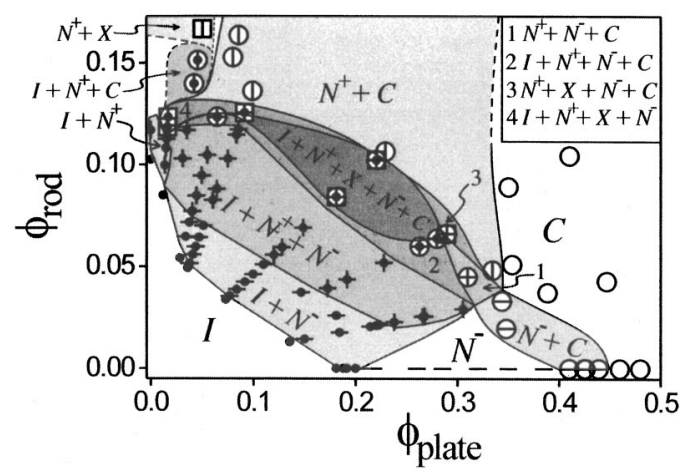

FIG. 7. Experimental phase diagram for mixtures of colloidal boehmite rods $\left(L_{r} / D_{r} \sim 10\right)$ and gibbsite platelets $\left(D_{p} / L_{p} \sim 15\right)$. Picture taken from Ref. 17. The phase behavior becomes considerably complicated beyond the triphasic area due to the presence of additional $N-C$ and $N-X$ transitions. tailed investigation of the lower part of the experimental phase diagram is probably required to detect this phenomenon.

The high concentration part of the experimental phase diagram is essentially different from the calculated one because additional high density liquid crystal phases (with partial positional order, such as the columnar $(C)$ phase and the not yet identified ${ }^{17}$ rod-rich phase $X$ ) come into play which are not taken into account theoretically. Most importantly, the theoretically predicted $N^{+}-N^{-}$demixing beyond the triphasic area is not observed experimentally. The absence of this equilibrium is probably caused by a combination of polydispersity and the presence of additional transitions from nematic to high-density liquid crystal phases $(C$ and $X)$. As a result, the $N^{+}-N^{-}$demixing is disrupted by several multiphase equilibria involving more than three phases, in particular, the remarkable five-phase equilibrium (see Fig. 7). This observation is very striking since it seems to contradict the Gibbs phase rule, which states that for an effective twocomponent system of hard particles only bi- and triphasic equilibria can be expected. A possible explanation for these phenomena is therefore the polydispersity of the colloidal species, i.e., the particles differ in size and shape. Since both species have a fairly high polydispersity (around 30\%) the resulting mixture effectively contains almost infinitely many components, which may lead to coexistence between arbitrarily many phases. Hence, the challenge raised for further theoretical research is not only to investigate the stability of high-density liquid crystal phases (e.g., columnar, smectic, etc.) but also to address the effect of polydispersity on the phase behavior of rod-plate mixtures.

\section{APPENDIX A}

In this Appendix, we give approximate analytical results for $\rho_{12}$ and $\rho_{22}$ by performing asymptotic expansions of the integrals.

\section{Excluded volume integral for particles with polar and equatorial orientation: $\rho_{12}$}

Inserting the Gaussian ODFs Eqs. (1), (2) into $\rho_{12}$, Eq. (10) yields

$$
\begin{aligned}
\rho_{12}= & \alpha_{1} \sqrt{\frac{\alpha_{2}}{(2 \pi)^{3}}} \int_{-\pi / 2}^{\pi / 2} \int_{-\pi / 2}^{\pi / 2} \int_{0}^{2 \pi}|\cos \gamma| \\
& \times \exp \left[-\frac{1}{2}\left(\alpha_{1} \theta^{2}+\alpha_{2} \psi^{2}\right)\right] d \phi d(\cos \theta) d(\sin \psi) .
\end{aligned}
$$

Here, $\theta$ is the polar angle (i.e., the angle between the particle axis and the $z$-axis) and $\psi=\pi / 2-\theta^{\prime}$ is the meridional angle between the particle vector and its projection onto the $x y$ plane. Furthermore, $\phi$ is the azimuthal angle between the projections of the particle vectors onto the $x y$ plane. Recall that the ODFs are sharply peaked around $\psi=0$ and $\theta=0$. Hence, using

$$
|\cos \gamma|=|\cos \theta \sin \psi+\sin \theta \cos \psi \cos \phi|,
$$

and substituting the asymptotic expressions, we can approximate 


$$
\begin{gathered}
\rho_{12} \sim 4 \alpha_{1} \sqrt{\frac{\alpha_{2}}{(2 \pi)^{3}}} \int_{0}^{\pi / 2} \int_{0}^{\pi / 2} \int_{0}^{2 \pi}|\psi+\theta \cos \phi| \\
\times \exp \left[-\frac{1}{2}\left(\alpha_{1} \theta^{2}+\alpha_{2} \psi^{2}\right)\right] d \phi \theta d \theta d \psi .
\end{gathered}
$$

In order to get rid of the absolute value sign in the integrand we must split the integral into parts. Noting that

$$
\begin{aligned}
& \psi+\theta \cos \phi<0 \text { if } \theta<\psi, \\
& \psi+\theta \cos \phi>0 \text { if } \psi<\theta \text { and if }-\phi_{\mathrm{G}}<\phi<\phi_{\mathrm{G}}, \\
& \psi+\theta \cos \phi<0 \text { if } \psi<\theta \text { and if }|\phi|>\phi_{\mathrm{G}}
\end{aligned}
$$

(with $\cos \phi_{\mathrm{G}}=-\psi / \theta$ ) we may split Eq. (A3) into three separate integrals

$$
\begin{aligned}
& \rho_{12} \sim 4 \alpha_{1} \sqrt{\frac{\alpha_{2}}{(2 \pi)^{3}}}\left\{\int_{0}^{\pi / 2} \int_{0}^{\pi / 2} \int_{-\pi}^{\pi}(\psi+\theta \cos \phi)\right. \\
& \times \exp \left[-\frac{1}{2}\left(\alpha_{1} \theta^{2}+\alpha_{2} \psi^{2}\right)\right] d \phi \theta d \theta d \psi \\
&-4 \int_{0}^{\pi / 2} \int_{\psi}^{\pi / 2} \int_{\phi_{\mathrm{G}}}^{\pi} \psi \exp \left[-\frac{1}{2}\left(\alpha_{1} \theta^{2}+\alpha_{2} \psi^{2}\right)\right] \\
& \times d \phi \theta d \theta d \psi-4 \int_{0}^{\pi / 2} \int_{\psi}^{\pi / 2} \int_{\phi_{\mathrm{G}}}^{\pi} \theta \cos \phi \\
&\left.\times \exp \left[-\frac{1}{2}\left(\alpha_{1} \theta^{2}+\alpha_{2} \psi^{2}\right)\right] d \phi \theta d \theta d \psi\right\}, \\
& \rho_{12} \sim 4 \alpha_{1} \sqrt{\frac{\alpha_{2}}{(2 \pi)^{3}}}\left\{I_{1}+I_{2}+I_{3}\right\} .
\end{aligned}
$$

Note that we can extend the integrations in $I_{1}$ with respect to $\theta$ and $\psi$ to infinity since the exponent decays rapidly to zero for large $\alpha_{1}$ and $\alpha_{2}$. The integral is then easily calculated and yields $I_{1}=2 \pi\left(\alpha_{1} \alpha_{2}\right)^{-1}$. For the second one, it is convenient to reverse the order of integration. Hence, we write

$$
\begin{aligned}
I_{2}= & -4 \int_{\pi / 2}^{\pi} \int_{0}^{\pi / 2} \int_{0}^{-\theta \cos \phi} \\
& \times \exp \left[-\frac{1}{2}\left(\alpha_{1} \theta^{2}+\alpha_{2} \psi^{2}\right)\right] \psi d \psi \theta d \theta d \phi \\
\sim & -4 \int_{\pi / 2}^{\pi} \int_{0}^{\infty} \exp \left(-\frac{1}{2} \alpha_{1} \theta^{2}\right) \alpha_{2}^{-1} \\
& \times\left[1-\exp \left(-\frac{1}{2} \alpha_{2} \theta^{2} \cos ^{2} \phi\right)\right] \theta d \theta d \phi .
\end{aligned}
$$

The integral can be worked out straightforwardly and yields $2 \pi\left(\alpha_{1} \alpha_{2}\right)^{-1}\left[\sqrt{\left[\alpha_{1} /\left(\alpha_{1}+\alpha_{2}\right)\right]}-1\right]$. For the third integral we use the relation $\int_{\phi_{\mathrm{G}}}^{\pi} \theta \cos \phi d \phi=-\sqrt{\theta^{2}-\psi^{2}}$ to write

$$
\begin{aligned}
I_{3}= & 4 \int_{0}^{\pi / 2} \int_{\psi}^{\pi / 2} \sqrt{\theta^{2}-\psi^{2}} \\
& \times \exp \left[-\frac{1}{2}\left(\alpha_{1} \theta^{2}+\alpha_{2} \psi^{2}\right)\right] \theta d \theta d \psi,
\end{aligned}
$$

substituting $y=\frac{1}{2} \alpha_{1}\left(\theta^{2}-\psi^{2}\right)$ yields

$$
\begin{aligned}
I_{3} \sim & 2^{5 / 2} \alpha_{1}^{3 / 2} \int_{0}^{\pi / 2} \exp \left[-\frac{1}{2}\left(\alpha_{1}+\alpha_{2}\right) \psi^{2}\right] d \psi \\
& \times \int_{0}^{\infty} y^{1 / 2} \exp (-y) d y \sim 2 \pi / \sqrt{\alpha_{1}^{3}\left(\alpha_{1}+\alpha_{2}\right)} .
\end{aligned}
$$

Finally, substituting all contributions back into Eq. (A5) thus yields for $\rho_{12}$

$$
\rho_{12} \sim \sqrt{\frac{8}{\pi}\left(\frac{1}{\alpha_{1}}+\frac{1}{\alpha_{2}}\right)}+\mathcal{O}\left(\alpha_{1}^{-3 / 2}, \alpha_{2}^{-3 / 2}\right),
$$

where the second term in the expansion can be shown to be of the order $\alpha_{j}^{-3 / 2}$.

\section{Excluded volume integral for particles with equatorial orientation: $\rho_{22}$}

Inserting the Gaussian ODFs Eqs. (1), (2) into $\rho_{22}$, Eq. (9) yields

$$
\begin{aligned}
\rho_{22}= & \frac{4}{\pi} \frac{\alpha_{2}}{(2 \pi)^{2}} \int_{-\pi / 2}^{\pi / 2} \int_{-\pi / 2}^{\pi / 2} \int_{0}^{2 \pi}|\sin \gamma| \\
& \times \exp \left[-\frac{\alpha_{2}}{2}\left(\psi^{2}+\psi^{\prime 2}\right)\right] d \phi d(\sin \psi) d\left(\sin \psi^{\prime}\right),
\end{aligned}
$$

which we can approximate, similar to $\rho_{12}$, as

$$
\begin{aligned}
\rho_{22} \sim & \frac{16}{\pi} \frac{\alpha_{2}}{(2 \pi)^{2}} \int_{0}^{\infty} \int_{0}^{\infty} K\left(\psi, \psi^{\prime}\right) \\
& \times \exp \left[-\frac{\alpha_{2}}{2}\left(\psi^{2}+\psi^{\prime 2}\right)\right] d \psi d \psi^{\prime} .
\end{aligned}
$$

Here, $K\left(\psi, \psi^{\prime}\right)$ is the azimuthally integrated kernel

$$
K\left(\psi, \psi^{\prime}\right) \equiv \int_{0}^{2 \pi}|\sin \gamma| d \phi=\int_{0}^{2 \pi} \sqrt{1-\cos ^{2} \gamma} d \phi .
$$

We can expand $K$ around $\psi=0$ since the particle vectors, on average, only marginally deviate from the $x y$ plane. Here, $\gamma$ is the angle between the two particle vectors $\mathbf{u}(\psi)$ and $\mathbf{u}^{\prime}(\psi, \phi)$,

$$
\mathbf{u}=\left(\begin{array}{c}
\cos \psi \\
0 \\
\sin \psi
\end{array}\right), \quad \mathbf{u}^{\prime}=\left(\begin{array}{c}
\cos \psi^{\prime} \cos \phi \\
\cos \psi^{\prime} \sin \phi \\
\sin \psi^{\prime}
\end{array}\right)
$$

Taking the square of the inner product of the two vectors and substituting the asymptotic expressions (up to second order in $\psi$ ) yields

$$
\begin{aligned}
\cos ^{2} \gamma & =\left(\cos \psi \cos \psi^{\prime} \cos \phi+\sin \psi \sin \psi^{\prime}\right)^{2} \\
& \approx\left[\left(1-\frac{1}{2} \psi^{2}\right)\left(1-\frac{1}{2} \psi^{\prime 2}\right) \cos \phi+\psi \psi^{\prime}\right]^{2} .
\end{aligned}
$$

Using cylindrical coordinates $\left(\psi=R \sin \chi\right.$ and $\left.\psi^{\prime}=R \cos \chi\right)$ and expanding up to second order in $R$ gives

$$
\cos ^{2} \gamma=\left(1-R^{2}\right) \cos ^{2} \phi+2 R^{2} \cos \phi \sin \chi \cos \chi+\mathcal{O}\left(R^{4}\right)
$$

and 


$$
\begin{aligned}
|\sin \gamma|= & \sqrt{1-\cos ^{2} \gamma} \\
\sim & \sqrt{1-\left(1-R^{2}\right) \cos ^{2} \phi} \\
& \times\left[1+\frac{R^{2} \cos \phi \sin \chi \cos \chi}{1-\left(1-R^{2}\right) \cos ^{2} \phi}+\cdots\right] .
\end{aligned}
$$

The kernel $K$ now reads ${ }^{29}$

$$
\begin{aligned}
K(R, \chi)= & \int_{0}^{2 \pi} \sqrt{1-\left(1-R^{2}\right) \cos ^{2} \phi} d \phi \\
& +\int_{0}^{2 \pi} \frac{R^{2} \cos \phi \sin \chi \cos \chi}{\sqrt{1-\left(1-R^{2}\right) \cos ^{2} \phi}} d \phi+\cdots
\end{aligned}
$$

The second integral is zero, since the integrand is an odd periodic function (with period $2 \pi$ ). Likewise, all higher contributions depending on odd powers of $\cos \phi$ are zero. The first integral in Eq. (A15) can be rewritten as the complete elliptic integral of the second kind $E(\kappa)$ via

$$
\begin{aligned}
\int_{0}^{2 \pi} \sqrt{1-\left(1-R^{2}\right) \cos ^{2} \phi} d \phi & =4 \int_{0}^{\pi / 2} \sqrt{1-\kappa \sin ^{2} \phi} d \phi \\
& =4 E(\kappa),
\end{aligned}
$$

where $\kappa=1-R^{2}$. This integral can be expanded around $R$ $=0$ up to second order ${ }^{30}$

$$
\begin{aligned}
E(\kappa) & =1+\frac{1}{2}\left[\ln \left(\frac{4}{R}\right)-\frac{1}{2}\right] R^{2}+\frac{3}{16}\left[\ln \left(\frac{4}{R}\right)-\frac{13}{12}\right] R^{4}+\ldots \\
& =1+\frac{1}{2}\left[\ln 4-\ln R-\frac{1}{2}\right] R^{2}+\mathcal{O}\left(R^{4}\right) .
\end{aligned}
$$

We now have the following expansion for the azimuthally integrated kernel:

$$
\begin{aligned}
K\left(\psi, \psi^{\prime}\right)= & 4+2\left[\ln 4-\frac{1}{2} \ln \left(\psi^{2}+\psi^{\prime 2}\right)-\frac{1}{2}\right]\left(\psi^{2}+\psi^{\prime 2}\right) \\
& +\mathcal{O}\left(\left\{\psi^{4} \ln \psi\right\}\right)
\end{aligned}
$$

valid for small $\psi$ and $\psi^{\prime}$. Substituting this into Eq. (A11) gives [in terms of the cylindrical coordinates $(\chi, R)]$

$$
\begin{aligned}
\rho_{22} \sim & \frac{32}{\pi} \frac{\alpha_{2}}{(2 \pi)^{2}} \int_{0}^{\infty} \int_{0}^{2 \pi}\left\{1+\frac{1}{2}\left[\ln 4-\ln R-\frac{1}{2}\right]\right\} \\
& \times \exp \left[-\frac{\alpha_{2}}{2} R^{2}\right] R d \chi d R
\end{aligned}
$$

which can be solved straightforwardly, yielding

$$
\begin{aligned}
\rho_{22} \sim & \frac{8}{\pi^{2}}\left[1+\frac{1}{2} \frac{\ln \alpha_{2}}{\alpha_{2}}+\frac{\ln (2 \sqrt{2})+\frac{1}{2} \gamma_{E}-1}{\alpha_{2}}\right] \\
& +\mathcal{O}\left(\alpha_{2}^{-2} \ln \alpha_{2}\right),
\end{aligned}
$$

where $\gamma_{E}=0.5772156649 \ldots$ denotes Euler's constant. Note that the $\ln \alpha_{2} / \alpha_{2}$ term is the leading order $\alpha_{2}$ depending term in this expansion.

The next step is to work out the higher order integrals contributing to the kernel Eq. (A15). It can be shown that these integrals give additional $\mathcal{O}\left(\alpha_{2}^{-1}\right)$ contributions to $\rho_{22}$. After tedious derivations we obtain the following final expression for $\rho_{22}$

$$
\begin{aligned}
\rho_{22} & \sim \frac{8}{\pi^{2}}\left[1+\frac{1}{2} \frac{\ln \alpha_{2}}{\alpha_{2}}+\frac{\ln 4+\frac{1}{2} \gamma_{E}-3 / 2}{\alpha_{2}}\right]+\mathcal{O}\left(\alpha_{2}^{-2} \ln \alpha_{2}\right) \\
& \sim \frac{8}{\pi^{2}}\left[1+\mathcal{F}\left(\alpha_{2}\right)\right]+\mathcal{O}\left(\alpha_{2}^{-2} \ln \alpha_{2}\right),
\end{aligned}
$$

which now contains all contributions up to order $\mathcal{O}\left(\alpha_{2}^{-1}\right)$.

\section{APPENDIX B}

Let $\alpha_{1,0}$ and $\alpha_{2,0}$ be the solutions of the minimization Eqs. (18) and (19) for the zeroth order problem $(\mathcal{F}=\mathcal{H}=0)$,

$$
\begin{aligned}
& \alpha_{1,0}^{1 / 2}=2 \pi^{-1 / 2}\left[(1-x)+2^{1 / 2} x q_{12} h\left(Q_{0}\right)\right] c, \\
& \alpha_{2,0}^{1 / 2}=2^{5 / 2} \pi^{-1 / 2} q_{12}(1-x) g\left(Q_{0}\right) c .
\end{aligned}
$$

When $\mathcal{F}$ is nonzero and $\mathcal{H}$ is given by Eq. (23) these solutions will only be marginally affected, since $\mathcal{H}$ is a small contribution. Hence, we can write

$$
\alpha_{1}=\alpha_{1}^{0}+\delta \alpha_{1}, \quad \alpha_{2}=\alpha_{2}^{0}+\delta \alpha_{2},
$$

where $\delta \alpha_{1}$ and $\delta \alpha_{2}$ are the perturbations, such that $\delta \alpha_{1} / \alpha_{1}^{0}$ and $\delta \alpha_{2} / \alpha_{2}^{0}$ are small parameters. We can linearize $Q\left(=\alpha_{2} / \alpha_{1}\right)$ with respect to these perturbation parameters

$$
Q=\frac{\alpha_{2}^{0}+\delta \alpha_{2}}{\alpha_{1}^{0}+\delta \alpha_{1}} \simeq Q_{0}\left[1+\frac{\delta \alpha_{2}}{\alpha_{2}^{0}}-\frac{\delta \alpha_{1}}{\alpha_{1}^{0}}\right],
$$

and, accordingly,

$$
\begin{aligned}
& g(Q) \simeq\left(1+Q_{0}\right)^{-1 / 2}\left[1-\frac{1}{2} \frac{Q_{0}}{Q_{0}+1}\left(\frac{\delta \alpha_{2}}{\alpha_{2}^{0}}-\frac{\delta \alpha_{1}}{\alpha_{1}^{0}}\right)\right], \\
& h(Q) \simeq\left(1+Q_{0}^{-1}\right)^{-1 / 2}\left[1+\frac{1}{2} \frac{1}{Q_{0}+1}\left(\frac{\delta \alpha_{2}}{\alpha_{2}^{0}}-\frac{\delta \alpha_{1}}{\alpha_{1}^{0}}\right)\right] .
\end{aligned}
$$

Similarly, we get

$$
\alpha_{1}^{1 / 2} \simeq \alpha_{1,0}^{1 / 2}\left[1+\frac{\delta \alpha_{1}}{2 \alpha_{1}^{0}}\right] \text { and } \alpha_{2}^{1 / 2} \simeq \alpha_{2,0}^{1 / 2}\left[1+\frac{\delta \alpha_{2}}{2 \alpha_{2}^{0}}\right] .
$$

To find explicit expressions for $\delta \alpha_{1}$ and $\delta \alpha_{2}$ we must substitute the above expressions into the minimization equations (with $\mathcal{H}$ nonzero). All zeroth order terms must cancel out, by construction, thus leaving an inhomogeneous set of linear equations in $\delta \alpha_{1}$ and $\delta \alpha_{2}$

$$
\begin{aligned}
& x \delta \alpha_{2}=\left[2(1-x)\left(1+Q_{0}\right)+Q_{0} x\right] \delta \alpha_{1}, \\
& \delta \alpha_{2}=-\frac{Q_{0}}{1+Q_{0}}\left[\delta \alpha_{2}-Q_{0} \delta \alpha_{1}\right]-2 \alpha_{2,0}^{1 / 2} c \mathcal{H}\left(x, \alpha_{2,0}\right),
\end{aligned}
$$

where the term depending on $\mathcal{H}$ is the inhomogeneous term ensuring nonzero solutions for $\delta \alpha_{1}$ and $\delta \alpha_{2}$. After solving the linear set we obtain explicit expressions for $\delta \alpha_{1}$ and $\delta \alpha_{2}$ (not shown here) which can be rewritten as functions of $Q_{0}(x), x$ and $c$, using Eq. (B1). It can be shown that the perturbations scale with concentration as $\delta \alpha_{j} \sim K_{j}(x) c$ $+c \ln c$, where $K_{j}(x)$ are functions of the mole fraction only. Hence, the perturbation $\mathcal{F}$ leads to an additional $c \ln c$ contribution (up to leading order) to the usual $c^{2}$-dependence of $\alpha_{1,0}$ and $\alpha_{2,0}$ Eq. (B1). 
The final step is to linearize the expressions $\sigma_{j}$ and $\rho_{j k}$. Substituting Eq. (B2) in Eqs. (3), (5) and expanding up to first order in $\delta \alpha_{1}$ and $\delta \alpha_{2}$ thus yields for the orientational entropy

$$
\begin{aligned}
& \sigma_{1}=\sigma_{1,0}+\delta \sigma_{1}=\ln \alpha_{1,0}-1+\frac{\delta \alpha_{1}}{\alpha_{1,0}}, \\
& \sigma_{2}=\sigma_{2,0}+\delta \sigma_{2}=\frac{1}{2}\left[\ln \alpha_{2,0}+\ln \frac{2}{\pi}-1\right]+\frac{\delta \alpha_{2}}{2 \alpha_{2,0}} .
\end{aligned}
$$

Similarly, we get for the excluded volume entropy

$$
\rho_{11}=\rho_{11,0}+\delta \rho_{11}=4 \pi^{-1 / 2}\left[\alpha_{1,0}^{-1 / 2}-\frac{\delta \alpha_{1}}{2 \alpha_{1,0}^{3 / 2}}\right],
$$

and

$$
\begin{aligned}
\rho_{12}= & \rho_{12,0}+\delta \rho_{12} \\
= & 2^{3 / 2} \pi^{-1 / 2}\left\{\left(\frac{1}{\alpha_{1,0}}+\frac{1}{\alpha_{2,0}}\right)^{1 / 2}\right. \\
& \left.-\left[\frac{h\left(Q_{0}\right) \delta \alpha_{1}}{2 \alpha_{1,0}^{3 / 2}}+\frac{g\left(Q_{0}\right) \delta \alpha_{2}}{2 \alpha_{2,0}^{3 / 2}}\right]\right\} .
\end{aligned}
$$

Obviously, the expression for $\rho_{22}$ Eq. (13) remains unchanged, since $\mathcal{F}$ constitutes the direct perturbation. Note that the leading order terms in Eqs. (B7), (B8) and (B9), (B10) correspond to the expressions for $\sigma_{j}$ and $\rho_{j k}$ given in Sec. II A. It is now straightforward to reexpress the free energy Eq. (16) of the nematic phase by simply adding perturbation terms depending on $\delta \sigma_{j}$ and $\delta \rho_{j k}$. Hence, we obtain the following perturbative contributions (denoted by $\delta$ ) to the osmotic pressure and chemical potentials of the nematic phase which must be added to the corresponding expressions given in Sec. II A:

$$
\begin{aligned}
& \delta \widetilde{\Pi}_{n} \sim-c\left[Q_{0}(1-x) \frac{\delta \alpha_{1}}{\alpha_{2,0}}+\frac{1}{2} x \frac{\delta \alpha_{2}}{\alpha_{2,0}}\right] \\
&+\frac{1}{2} c x \mathcal{W}\left(x, c, Q_{0}\right), \\
& \delta \widetilde{\mu}_{1, n} \sim \frac{x}{2(1-x)}\left[Q_{0}^{2} \frac{\delta \alpha_{1}}{\alpha_{2,0}}-\frac{\delta \alpha_{2}}{\alpha_{2,0}}\right]-Q_{0} \frac{\delta \alpha_{1}}{\alpha_{2,0}}, \\
& \delta \widetilde{\mu}_{2, n} \sim-\frac{1}{2} Q_{0}^{2} \frac{\delta \alpha_{1}}{\alpha_{2,0}}+\mathcal{W}\left(x, c, Q_{0}\right),
\end{aligned}
$$

with

$$
\mathcal{W}\left(x, c, Q_{0}\right)=\frac{1}{4 \pi c} \frac{x q_{22}}{q_{12}^{2}(1-x)^{2}}\left(1+Q_{0}\right)\left(\ln \alpha_{2,0}+2 K\right) .
$$

Note that the terms depending on $\mathcal{W}$ are the direct perturbations (arising from $\delta \rho_{22}$ ).

${ }^{1}$ H. Zocher, Z. Anorg. Allg. Chem. 147, 91 (1925).

${ }^{2}$ I. Langmuir, J. Chem. Phys. 6, 873 (1938).

${ }^{3}$ J. D. Bernal and I. Fankuchen, J. Gen. Physiol. 25, 111 (1941).

${ }^{4}$ M. P. B. van Bruggen, F. M. van der Kooij, and H. N. W. Lekkerkerker, J. Phys.: Condens. Matter 8, 9451 (1996).

${ }^{5}$ F. M. van der Kooij and H. N. W. Lekkerkerker, J. Phys. Chem. B 102, 7829 (1998)

${ }^{6}$ D. Frenkel and R. Eppenga, Phys. Rev. Lett. 49, 1089 (1982).

${ }^{7}$ L. Onsager, Ann. N.Y. Acad. Sci. 51, 627 (1949).

${ }^{8}$ R. Alben, J. Chem. Phys. 59, 4299 (1973).

${ }^{9}$ A. Saupe, P. Boonbrahm, and L. J. Yu, J. Chim. Phys. 80, 7 (1983).

${ }^{10}$ Y. Rabin, W. E. M. Mullen, and W. M. Gelbart, Mol. Cryst. Liq. Cryst. 89, 67 (1982).

${ }^{11}$ A. Chrzanowska, Phys. Rev. E 58, 3229 (1998).

${ }^{12}$ A. Stroobants and H. N. W. Lekkerkerker, J. Phys. Chem. 88, 3669 (1984).

${ }^{13}$ P. J. Camp and M. P. Allen, Physica A 229, 410 (1996).

${ }^{14}$ R. van Roij and B. Mulder, J. Phys. II 4, 1763 (1994).

${ }^{15}$ P. J. Camp, M. P. Allen, P. G. Bolhuis, and D. Frenkel, J. Chem. Phys. 106, 9270 (1997).

${ }^{16}$ F. M. van der Kooij and H. N. W. Lekkerkerker, Phys. Rev. Lett. 84, 781 (2000).

${ }^{17}$ F. M. van der Kooij and H. N. W. Lekkerkerker, Langmuir 16, 10144 (2000).

${ }^{18}$ J. A. C. Veerman and D. Frenkel, Phys. Rev. A 45, 5632 (1992).

${ }^{19}$ D. Frenkel, J. Phys. Chem. 91, 4912 (1987).

${ }^{20}$ J. D. Parsons, Phys. Rev. A 19, 1225 (1979).

${ }^{21}$ S. D. Lee, J. Chem. Phys. 89, 7036 (1989).

${ }^{22}$ D. Frenkel, H. N. W. Lekkerkerker, and A. Stroobants, Nature (London) 332, 822 (1988).

${ }^{23}$ T. Odijk and H. N. W. Lekkerkerker, J. Phys. Chem. 89, 2090 (1985).

${ }^{24}$ R. van Roij and B. Mulder, J. Chem. Phys. 105, 11237 (1996).

${ }^{25}$ G. J. Vroege and H. N. W. Lekkerkerker, J. Phys. Chem. B 97, 3601 (1993).

${ }^{26}$ J. P. Hansen and I. R. McDonald, Theory of Simple Liquids (Academic, London, 1986).

${ }^{27}$ P. Bartlett, J. Phys.: Condens. Matter 2, 4979 (1990).

${ }^{28}$ H. N. W. Lekkerkerker, P. Coulon, R. van der Hagen, and R. Deblieck, J. Chem. Phys. 80, 3427 (1984).

${ }^{29}$ The higher order terms denoted by ... involve elliptic integrals of the form $\int_{0}^{2 \pi} d \phi R^{4} \cos ^{2} \phi / \sqrt{1-\left(1-R^{2}\right) \cos ^{2} \phi} \quad$ and $\int_{0}^{2 \pi} d \phi R^{4} \cos ^{2} \phi /[1-(1$ $\left.\left.-R^{2}\right) \cos ^{2} \phi\right]^{3 / 2}$ which can be rewritten in terms of complete elliptic integrals of the second and third kind (Ref. 30).

${ }^{30}$ P. F. Byrd and M. D. Friedman, Handbook of Elliptic Integrals for Engineers and Physicists (Springer-Verlag, Berlin, 1954). 